\title{
Measurement of top quark properties at $\sqrt{s}=1.96 \mathrm{TeV}$ using the $\mathrm{D} \varnothing$ detector
}

\author{
Alexander Grohsjean* on behalf of the DØ Collaboration \\ Irfu/SPP/CEA Saclay \\ E-mail: agrohsjedfnal.gov
}

\begin{abstract}
Since the discovery of the top quark in 1995 at the Fermilab Tevatron collider, top quark properties have been measured with ever higher precision. The increase in data has provided the opportunity to probe many new aspects of the top quark sector. In addition to an updated measurement of the helicity of the $W$ boson in $t \bar{t}$ events using $5.4 \mathrm{fb}^{-1}$, this review briefs three new analyses performed at the $\mathrm{D} \emptyset$ experiment: a measurement of spin correlation in dilepton $t \bar{t}$ decays based on angular distributions, a second using a matrix-element approach, and finally a study of color flow in lepton+jets final states.
\end{abstract}

The 2011 Europhysics Conference on High Energy Physics-HEP 2011,

July 21-27, 2011

Grenoble, Rhône-Alpes France

\footnotetext{
* Speaker.
} 


\section{Introduction}

With a mass of $173.2 \pm 0.9 \mathrm{GeV}$ [1], the top quark is the heaviest of all known fundamental particles. The top quark Yukawa coupling is close to unity, suggesting that it may play a special role in electroweak symmetry breaking [2]. With a predicted lifetime of about $10^{-25} \mathrm{~s}$, the top quark decays through the electroweak interaction before forming hadrons [3, 4]. Thus, it is the only quark that provides a direct probe of the properties of a bare quark.

At the Tevatron $p \bar{p}$ collider with a center-of-mass energy of $1.96 \mathrm{TeV}, 85 \%$ of the $t \bar{t}$ pairs are produced through quark-antiquark annihilation, while $15 \%$ originate from gluon-gluon fusion. In the standard model (SM), top quarks are predicted to decay almost exclusively to a $W$ boson and a bottom quark, and $t \bar{t}$ events can therefore be classified into all-jets, lepton+jets and dilepton events depending on the modes of the $W$ decays. The lepton+jets channel [5] is characterized by the presence of four jets, one isolated, energetic charged lepton and an imbalance in transverse momentum. The $30 \%$ branching fraction of $t \bar{t}$ events into lepton+jets channels, where lepton can be either an electron or muon, corresponds to a factor of about six more events than for the cleaner dilepton channels whereas the signal to background ratio is about a factor of three smaller. The main background in lepton+jets is from $W+$ jets events. Instrumental background arises from events in which a jet is misidentified as a lepton and events with heavy quarks that decay into leptons that pass isolation requirements. The topology of the dilepton channel [6] is described by two jets, two isolated, energetic charged leptons and a significant imbalance in transverse momentum from the undetected neutrinos. Here, the main background processes are $Z+$ jets and diboson events $(W W$, $W Z$ and $Z Z$ with associated jets), as well as the kind of instrumental background characterized above.

To make use of the top quark in searches for new physics, it is mandatory to understand whether it represents really the particle predicted by the SM or not. In particular, if, for example, the lifetime, the decay properties or spin correlation deviate from the expected values, this could point to physics beyond the SM.

\section{Measurement of spin correlations in $t \bar{t}$ events}

With the increased data recorded at the $\mathrm{D} \emptyset$ experiment and the different nature of $t \bar{t}$ production at the Tevatron (85\% incident $q \bar{q}$ ) compared to the LHC (90\% incident $g g$ ), measuring spin correlation in $t \bar{t}$ decays has become one of the flagships of the Tevatron top-quark program. This measurement is of particular interest, as it tests the full chain from strong production to the electroweak decay of the top quarks. Deviations from the SM prediction could arise from additional contributions in production like e.g. from stop-quark pairs as well as from new decay modes of the top quark, such as to a charged Higgs boson $\left(t \rightarrow H^{+} b\right)$. Moreover, if the lifetime of the top quark is much longer than expected, the spin could flip through final-state interactions before decay resulting in a reduction of the spin correlation. Thus, the nature of $t \bar{t}$ spin correlation can also be influenced by the lifetime of the top quark.

Although top quarks are not produced in a polarized state at hadron colliders, their spins are 
correlated. The spin correlation strength $A$ in production is defined as

$$
A=\frac{N_{\uparrow \uparrow}+N_{\downarrow \downarrow}-N_{\uparrow \downarrow}-N_{\downarrow \uparrow}}{N_{\uparrow \uparrow}+N_{\downarrow \downarrow}+N_{\uparrow \downarrow}+N_{\downarrow \uparrow}}
$$

where $A$ reflects the number of events with spins pointing in the same as opposed to opposite directions [7]. The correlation strength $A$ is different for $t \bar{t}$ events produced via quark-antiquark annihilation and via gluon-gluon fusion. Its value depends on the choice of quantization axis, one of which is defined relative to the direction of the incoming proton and antiproton. This socalled beam basis is best suited for top quarks produced at threshold, and provides almost the maximum correlation strength. In next-to-leading-order quantum chromodynamics (NLO QCD), $A=0.78 \pm 0.04$. As the lifetime of the top quark is expected to be less than $\frac{1}{\Lambda_{\mathrm{QCD}}}$, its spin does not flip before decaying and $A$ can therefore be inferred from the angular distributions of the decay products. With $\theta$ defining the angle between and the beam axis and the final state particle in the $t$ $(\bar{t})$ rest frame, one derives

$$
\frac{1}{\sigma} \frac{d \sigma}{d \cos (\theta)}=\frac{1}{2}(1+\alpha \cos (\theta))
$$

The so-called spin analyzing power $\alpha$ depends on the final-state particle, and it is largest for charged leptons ( $\alpha=1.0$ in NLO QCD) and down-type quarks ( $\alpha=0.97$ in NLO QCD).

Putting all this together, the spin correlation strength can be measured by studying the double differential cross section

$$
\frac{1}{\sigma} \frac{d^{2} \sigma}{d \cos \left(\theta_{1}\right) d \cos \left(\theta_{2}\right)}=\frac{1}{4}\left(1-C \cos \left(\theta_{1}\right) \cos \left(\theta_{2}\right)\right)
$$

where $C=A \alpha_{1} \alpha_{2}$.

Thus, despite the small branching fraction of the $W \rightarrow \ell v_{\ell}$ decays, the dilepton channel is best for measuring spin correlation in $t \bar{t}$ decays, because unlike down-type quarks, charged leptons can be easily identified. In addition, the momentum of leptons is well measured and the contamination from background in this final state is very small.

To study the differential cross section of Eq. (2.3), templates have to be formed for the products of $\cos \left(\theta_{1}\right) \cos \left(\theta_{2}\right)$, that require a full reconstruction of the angles of the charged leptons in the $t(\bar{t})$ rest frame. One approach is the so-called neutrino-weighting method [8], where both neutrino pseudorapidities are sampled from expected Monte Carlo (MC) distributions. For each point in phase space, all solutions are weighted according to the agreement of the reconstructed neutrino momenta with the measured imbalance in transverse momentum. The weighted mean of all kinematic solutions is used to estimate $\cos \left(\theta_{1}\right) \cos \left(\theta_{2}\right)$.

The spin correlation strength $C$ is extracted from a binned maximum likelihood fit. Signal templates obtained from MC@NLO [9], with and without spin correlations, are mixed together as a function of $C$, including contributions from background. Systematic uncertainties are incorporated into the fit as free parameters, and the ordering principle for ratios of likelihoods [10] is used to set limits or extract a central value.

Based on a total of 441 dilepton candidate events, with an expected signal purity of $74 \%$, the spin correlation strength $C$ is extracted to be

$$
\begin{aligned}
C & =0.10 \pm 0.45 \text { (stat }+ \text { syst }) \\
-0.66 & <C<0.81 \text { at } 95 \% \text { C.L. }
\end{aligned}
$$


This is consistent within two standard deviations with the NLO prediction of $C=0.78 \pm 0.04$. The measurement is dominated by a statistical uncertainly of about 0.4 , with the largest systematic uncertainty being 0.07 resulting from limited statistics of the MC samples used to form the templates.

An increased sensitivity to spin correlation can be reached by employing a matrix-element approach [11] as widely used, for example, in top quark mass measurements. For each observed final state $x$, the probability that it arises from $q \bar{q} \rightarrow t \bar{t}$ production with spins correlated according to the $\mathrm{SM}(\mathrm{H}=\mathrm{c})$, or uncorrelated spins $(\mathrm{H}=\mathrm{u})$ is given by:

$$
P_{s g n}(x ; H) \propto \int d \varepsilon_{1} d \varepsilon_{2} f_{P D F}\left(\varepsilon_{1}\right) f_{P D F}\left(\varepsilon_{2}\right) \frac{|M(y ; H)|^{2}}{\varepsilon_{1} \varepsilon_{2} s} W(x, y) d \Phi_{6},
$$

where $\varepsilon_{1}, \varepsilon_{2}$ are the energy fractions of the incoming partons, $f_{P D F}$ are the leading-order parton distribution functions CTEQ6L1 [12], $M(y ; H)$ is the leading-order matrix element [13] and $d \Phi_{6}$ is an element of the six-body production phase space. The transfer functions $W(x, y)$ reflect the probability on a partonic object $y$ to be reconstructed as an observed object $x$.

Based on these probabilities, we can define a variable

$$
R=\frac{P_{s g n}(H=c)}{P_{s g n}(H=c)+P_{s g n}(H=u)}
$$

to discriminate between $t \bar{t}$ events with $(c)$ and without $(u)$ SM spin correlation [14]. As in the measurement based on the $\cos \left(\theta_{1}\right) \cos \left(\theta_{2}\right)$ templates, $C$ is extracted from a binned maximumlikelihood fit using signal templates in $R$ from MC@NLO with and without spin correlations as well as contributions from background. Systematic uncertainties are again incorporated as free parameters in the fit.

From a total of 485 dilepton candidate events with an expected signal purity of about $71 \%$, the spin correlation strength $C$ is measured to be

$$
\begin{aligned}
& C=0.57 \pm 0.31(\text { stat }+ \text { syst }) \\
& C>0.11 \text { at } 95 \% \text { C.L. }
\end{aligned}
$$

which is consistent with the NLO prediction of $C=0.78 \pm 0.04$. The improved sensitivity of about $30 \%$ of this analysis makes this the first measurement that can exclude the case of no spin correlation $C=0$ at the $97.7 \%$ C.L., where a confidence of $99.6 \%$ is expected from MC simulation. The largest systematic uncertainty of 0.07 is again due to the limited statistics available in forming the templates.

\section{Measurement of the $W$ boson helicity}

In the SM, the coupling of the $W$ boson to fermions is predicted to be mainly left-handed. Thus, positive helicity states of the $W$ boson are highly suppressed, while the fraction of longitudinal helicity states dominates with a fraction of $f_{0}=0.7$ [15]. Any significant deviation from the SM expectation can be an indication of new physics arising either from a deviation from the expected $V-A$ coupling of the $t \rightarrow W b$ vertex or the presence of non-SM contributions in the data. 
A very powerful variable to distinguish between different helicity states is the opening angle $\cos \left(\theta^{*}\right)$ between the down-type fermion from the $W$ boson decay and the negative top quark momentum in the rest frame of the $W$ boson [16]. The calculation of $\cos \left(\theta^{*}\right)$ requires full $t \bar{t}$ event reconstruction. In both, the dilepton and the lepton+jets channel, the top quark mass and the $W$ boson mass are assumed to be known and the total $t \bar{t}$ transverse momentum is set to the opposite of the unclustered energy in the calorimeter. As the down-type quark from the hadronic $W$ decay in lepton+jets events cannot be identified reliably, one jet from the $W$ decay is chosen randomly as the evolved down quark, and the absolute value $\left|\cos \left(\theta^{*}\right)\right|$ is used to separate $f_{0}$ from $f_{+}$and $f_{-}$contributions. To derive templates for each state of $W$ helicity, $\cos \left(\theta^{*}\right)$ is calculated for pure $V-A$ and $V+A$ possibilities and combined to provide left-handed, longitudinal and right-handed helicity states.

From a total luminosity of $5.4 \mathrm{fb}^{-1}$ of lepton+jets and dilepton events, the $W$ boson helicity fractions $f_{0}$ and $f_{+}$are extracted simultaneously performing a binned Poisson likelihood fit to obtain:

$$
\begin{aligned}
& f_{0}=0.669 \pm 0.078(\text { stat }) \pm 0.065(\text { syst }) \\
& f_{+}=0.023 \pm 0.041(\text { stat }) \pm 0.034(\text { syst }) .
\end{aligned}
$$

Both values are in agreement with SM expectation. The largest systematic uncertainty on $f_{0}$ comes from the $t \bar{t}$ modeling, estimated to be 0.033 . This is the first time that the systematic and statistical uncertainties on $f_{0}$ are of about the same size.

\section{Measurement of color flow in $t \bar{t}$ events}

QCD color charge is conserved locally and flows like electrical charge. Pulling apart color from its anticolor component forms color connections. The pairing of connections depends thereby on the nature of the decaying particle. In case of color-neutral objects, color singlets, such as Higgs or $W$ bosons, the decay particles are color connected to each other. For color octets, e.g., gluons, the decay particles are color connected to the beam remnant. The breaking of color connections produces hadrons and the consequent color flow modifies the characteristics of the jet shapes. Thus, analyzing color flow can be a a powerful tool to search for new physics as it helps to separate different processes within some given final states, such as $Z H \rightarrow Z b \bar{b}$ and background from $Z+$ jets.

A variable well suited to describe the color flow, is the "jet pull" variable [17], which is given by

$$
\vec{p}=\sum_{i}^{n_{\text {cells }}} \frac{E_{T}^{i}\left|r_{i}\right|}{E_{T}^{\mathrm{jet}}} \vec{r}_{i},
$$

where $\vec{r}_{i}$ is the position of cell $i$ in the calorimeter relative to the jet axis, $E_{T}^{i}$ the transverse energy deposited in the cell and $E_{T}^{\text {jet }}$ the total transverse energy of the jet. Thus, the jet pull vectors tend to point towards each other for jets originating from color singlets compared to jets from color octets.

Before the jet pull can be applied in searches for new physics, this tool has to be studied under known conditions such as, e.g., lepton+jets $t \bar{t}$ events, where both light-quark jets from $W$ boson decay are expected to arise from a color singlet [18]. Using $5.3 \mathrm{fb}^{-1}$ of lepton+jets data, the events are compared to SM $t \bar{t}$ MC events with a color-singlet $W$ boson and to a $t \bar{t}$ MC sample with a 
hypothetical color-octet $W$ boson. The difference between the two MC samples is greatest in the central region of the detector, when both light-quark jets are close to each other in space and their invariant mass is close to the mass of the $W$ boson. From a total of 728 lepton+jets candidate events, the fraction of events fitted to a color-singlet $W$-boson contribution in a binned maximum likelihood fit, including systematic uncertainties as free parameters, is found to be

$$
f_{\text {singlet }}=0.56 \pm 0.38(\text { stat }+ \text { syst }) \pm 0.19(\text { MC stat }),
$$

which is consistent with the SM expectation of $f_{\text {singlet }}=1$. The largest systematic uncertainty on this measurement arises from the uncertainty on the MC modeling of the jet pull, as well as the inner cryostat region in the calorimeter. However, the current uncertainty is dominated largely by statistics.

\section{Summary}

With increased data at the Tevatron $p \bar{p}$ collider, essential properties of the top quark are being measured with higher precision or probed for the first time. Three of these measurements are reviewed briefly in this report, corresponding to a measurement of the helicity fractions of the $W$ bosons from top quark decays and two measurements of $t \bar{t}$ spin correlation. All analyzes were based on about $5.4 \mathrm{fb}^{-1}$ of data, and are in good agreement with the SM. In addition, well-understood $t \bar{t}$ events are used to explore color flow as a tool in the search for new physics.

\section{References}

[1] The Tevatron Electroweak Working Group, arXiv:1107.5255 [hep-ex] (2011).

[2] M. Hashimoto, M. Tanabashi and K. Yamawaki, Phys. Rev. D 64, 056003 (2001).

[3] I. I. Y. Bigi et al., Phys. Lett. B 181, 157 (1986).

[4] V. M. Abazov et al. (DØ Collaboration), Phys. Rev. Lett. 106, 022001 (2011).

[5] V. M. Abazov et al. (DØ Collaboration), Phys. Rev. D 84, 012008 (2011).

[6] V. M. Abazov et al. (DØ Collaboration), Phys. Lett. B 704, 403 (2011).

[7] W. Bernreuther et al., Nucl. Phys. B 690, 81 (2004). A. Brandenburger et al., Phys. Lett. B 539, 235 (2002).

[8] V. M. Abazov et al. (DØ Collaboration), Phys. Lett. B 702 ,16 (2011).

[9] S. Frixione and B. R. Webber, J. High Energy Phys. 06, 029 (2002).

[10] G. J. Feldman and R. D. Cousins, Phys. Rev. D 57, 3873 (1998).

[11] V. M. Abazov et al. (DØ Collaboration), Phys. Rev. Lett. 107, 032001 (2011).

[12] J. Pumplin et al. (CTEQ Collaboration), J. High Energy Phys. 07, 012 (2002).

[13] G. Mahlon and S. J. Parke, Phys. Rev. D 53, 4886 (1996); G. Mahlon and S. J. Parke, Phys. Lett. B 411, 173 (1997).

[14] K. Melnikov and M. Schulze, Phys. Lett. B 700, 17 (2011). 
[15] M. Fischer et al., Phys. Rev. D 63, 031501 (2001).

[16] V. M. Aabzov et al. (DØ Collaboration), Phys. Rev. D 83, 032009 (2011).

[17] Gallicchio et al., Phys. Rev. Lett. 105, 022001 (2011).

[18] V. M. Abazov et al. (DØ Collaboration), Phys. Rev. D 83, 092002 (2011). 